\title{
Chapter 80 \\ Report on the Global Environment \\ Competitiveness of Kyrgyzstan
}

Kyrgyzstan is located in the northeast of Central Asia, borders on China, Kazakhstan, Uzbekistan, Tajikistan. It is continental climate, and the deposit of coal, mercury and antimony are rich. It covers 192 thousand square kilometres. It had a population of 5.5 million and domestic production the gross (GDP) of USD5.9 billion in 2011. Through the evaluation of global environment competitiveness, we can know that the environment competitiveness index of Kyrgyzstan ranks at 123 in 133 countries.

Fig. 80.1 Contribution of

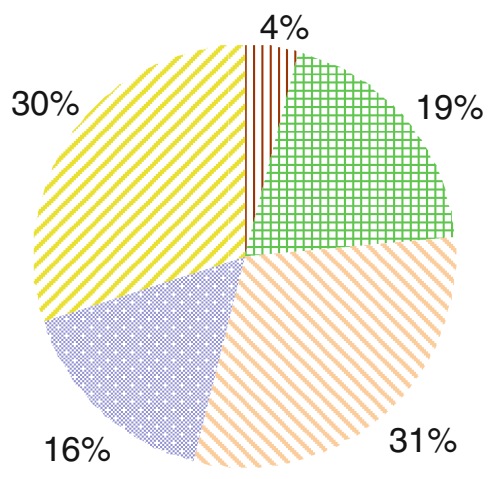

III Resource Environment Competitiveness

\# Ecological Environment Competitiveness

Environment Carrying Competitiveness

* Environment Management Competitiveness sub-index of GEC Environment Harmony Competitiveness 


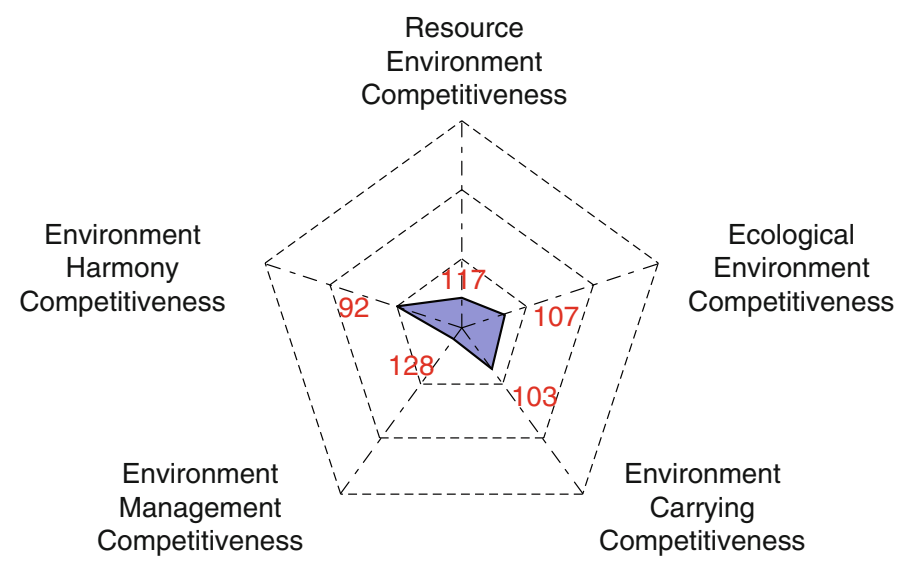

Fig. 80.2 Rank of sub-index of GEC

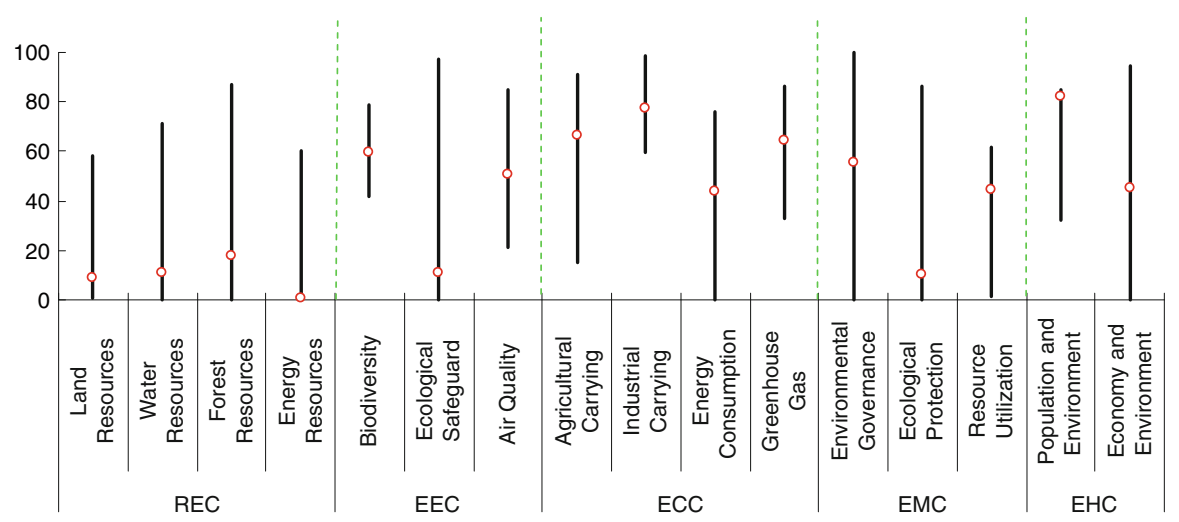

Fig. 80.3 Score and rank of the pillars of GEC

Table 80.1 Score and rank of all indicators of GEC

\begin{tabular}{|c|c|c|c|c|c|}
\hline Indicators & Score & Rank & Indicators & Score & Rank \\
\hline $\begin{array}{l}1 \text { Resource Environment } \\
\text { Competitiveness }\end{array}$ & 8.98 & 117 & $\begin{array}{l}\text { Total internal renewable } \\
\text { water resources }\end{array}$ & 12.87 & 70 \\
\hline 1.1 Land Resources & 9.09 & 90 & 1.3 Forest Resources & 17.78 & 110 \\
\hline Land area per capita & 6.25 & 32 & Growing stock in forest and & 50.14 & 99 \\
\hline $\begin{array}{l}\text { Percentage of arable land to } \\
\text { total land area }\end{array}$ & 11.14 & 95 & $\begin{array}{c}\text { other wooded land } \\
\text { Proportion of land area }\end{array}$ & 5.93 & 110 \\
\hline Arable land per capita & 10.83 & 53 & covered by forest & & \\
\hline 1.2 Water Resources & 11.15 & 86 & Forest area per capita & 1.23 & 78 \\
\hline Surface water & 2.65 & 81 & 1.4 Energy Resources & 0.86 & 117 \\
\hline Annual precipitation & 18.85 & 98 & Fossil energy & 0.00 & 64 \\
\hline Groundwater & 10.24 & 66 & Energy production & 0.22 & 113 \\
\hline
\end{tabular}


Table 80.1 (continued)

\begin{tabular}{|c|c|c|c|c|c|}
\hline Indicators & Score & Rank & Indicators & Score & Rank \\
\hline \multirow{2}{*}{$\begin{array}{l}\text { Proportion of combustible } \\
\text { renewables and waste to } \\
\text { total energy } \\
\text { consumption }\end{array}$} & \multirow[t]{2}{*}{0.13} & \multirow[t]{2}{*}{110} & $\begin{array}{l}\text { Energy consumption per } \\
\text { unit of land area }\end{array}$ & 99.97 & 17 \\
\hline & & & $\begin{array}{l}\text { Ratio of clean energy } \\
\text { consumption }\end{array}$ & 48.30 & 9 \\
\hline $\begin{array}{l}\text { Net energy imports of the } \\
\text { energy consumption }\end{array}$ & 5.04 & 98 & $\begin{array}{l}\text { Elasticity of energy } \\
\text { consumption }\end{array}$ & 15.66 & 15 \\
\hline $\begin{array}{l}2 \text { Ecological Environment } \\
\text { Competitiveness }\end{array}$ & 41.28 & 107 & $\begin{array}{l}\text { Elasticity of electric power } \\
\text { consumption }\end{array}$ & 12.38 & 30 \\
\hline 2.1 Biodiversity & 59.34 & 27 & 3.4 Greenhouse Gas & 64.11 & 48 \\
\hline Threatened fish species & 98.58 & 9 & \multirow{2}{*}{$\begin{array}{l}\text { Growth rate of } \mathrm{CO}_{2} \\
\text { emissions }\end{array}$} & \multirow[t]{2}{*}{67.85} & \multirow[t]{2}{*}{22} \\
\hline Threatened mammal species & 96.74 & 30 & & & \\
\hline Threatened plant species & 99.18 & 70 & Growth rate of Methane & \multirow[t]{2}{*}{49.33} & \multirow[t]{2}{*}{116} \\
\hline GEF benefits index for & 1.10 & 81 & \multirow{2}{*}{$\begin{array}{l}\text { emissions } \\
\mathrm{CO}_{2} \text { emissions per unit of } \\
\quad \text { land area }\end{array}$} & & \\
\hline $\begin{array}{l}\text { biodiversity } \\
\text { 2.2 Ecological Safeguard }\end{array}$ & 11.09 & 94 & & 99.96 & 32 \\
\hline Terrestrial protected areas & 18.48 & 84 & \multirow{2}{*}{$\begin{array}{l}\mathrm{CO}_{2} \text { emissions per unit of } \\
\text { energy consumption }\end{array}$} & \multirow[t]{2}{*}{35.57} & \multirow[t]{2}{*}{87} \\
\hline Marine protected areas & N/A & N/A & & & \\
\hline 2.3 Air Quality & 50.37 & 95 & \multirow{2}{*}{$\begin{array}{l}4 \text { Environment Management } \\
\text { Competitiveness }\end{array}$} & \multirow{2}{*}{33.98} & \multirow[t]{2}{*}{128} \\
\hline Inhalable particles (PM10) & 74.45 & 79 & & & \\
\hline Particulate matter (PM2.5) & 73.90 & 101 & 4.1 Environmental & 55.20 & 125 \\
\hline $\begin{array}{l}\text { Index of indoor air } \\
\text { pollution }\end{array}$ & 14.20 & 86 & $\begin{array}{l}\text { Governance } \\
\text { Agricultural chemicals }\end{array}$ & 0.00 & 86 \\
\hline Nitrogen oxides emission & 68.69 & 32 & regulation & \multirow{3}{*}{85.00} & \multirow{3}{*}{72} \\
\hline Sulfur dioxide emission & 40.92 & 26 & \multirow{2}{*}{$\begin{array}{l}\text { Percentage of the rural } \\
\text { population with access } \\
\text { to an improved water } \\
\text { source }\end{array}$} & & \\
\hline $\begin{array}{l}3 \text { Environment Carrying } \\
\text { Competitiveness }\end{array}$ & 64.62 & 103 & & & \\
\hline 3.1 Agricultural Carrying & 66.17 & 69 & \multirow{3}{*}{$\begin{array}{l}\text { Percentage of the urban } \\
\text { population with access } \\
\text { to an improved water } \\
\text { source }\end{array}$} & \multirow{3}{*}{99.00} & \multirow{3}{*}{51} \\
\hline $\begin{array}{l}\text { Cereal yield per unit of } \\
\text { arable land }\end{array}$ & 25.19 & 77 & & & \\
\hline $\begin{array}{l}\text { Fertilizer consumption per } \\
\text { unit of arable land }\end{array}$ & 98.30 & 38 & & & \\
\hline \multirow{2}{*}{$\begin{array}{l}\text { Annual freshwater } \\
\text { withdrawals for } \\
\text { agriculture per unit of } \\
\text { arable land }\end{array}$} & \multirow[t]{2}{*}{88.69} & \multirow[t]{2}{*}{113} & $\begin{array}{l}\text { 4.2 Ecological Protection } \\
\text { Area of plantation and } \\
\text { afforestation }\end{array}$ & $\begin{array}{r}10.03 \\
0.07\end{array}$ & $\begin{array}{r}123 \\
93\end{array}$ \\
\hline & & & Biome protect & 23.30 & 105 \\
\hline 3.2 Industrial Carrying & 77.08 & 127 & Overfishing of fishing & N/A & N/A \\
\hline $\begin{array}{l}\text { Net exports as a percentage } \\
\text { of GDP }\end{array}$ & 78.84 & 78 & 4.3 Resource Utilization & 44.69 & 30 \\
\hline $\begin{array}{l}\text { Electric power consumption } \\
\text { per unit of value added }\end{array}$ & 54.70 & 124 & $\begin{array}{l}\text { Utilization rate of water } \\
\text { resources }\end{array}$ & 1.77 & 21 \\
\hline of industry & & & Percentage of total internal & 80.60 & 36 \\
\hline $\begin{array}{l}\mathrm{SO}_{2} \text { emissions per unit of } \\
\text { value added of industry }\end{array}$ & 99.37 & 107 & $\begin{array}{l}\text { renewable water } \\
\text { resources to total water } \\
\text { resources }\end{array}$ & & \\
\hline $\begin{array}{l}\text { Annual freshwater } \\
\text { withdrawals for industry }\end{array}$ & 75.43 & 125 & $\begin{array}{l}\text { Percentage of agricultural } \\
\text { land to total land area }\end{array}$ & 65.43 & 41 \\
\hline & & & Percentage of fossil fuel & 30.95 & 53 \\
\hline 3.3 Energy Consumption & 44.08 & 13 & total energy consumption & & \\
\hline
\end{tabular}


Table 80.1 (continued)

\begin{tabular}{|c|c|c|c|c|c|}
\hline Indicators & Score & Rank & Indicators & Score & Rank \\
\hline $\begin{array}{l}5 \text { Environment Harmony } \\
\text { Competitiveness }\end{array}$ & 63.78 & 92 & $\begin{array}{l}\mathrm{CO}_{2} \text { emissions (metric tons } \\
\text { per capita) }\end{array}$ & 96.58 & 40 \\
\hline $\begin{array}{l}\text { 5.1 Population and } \\
\text { Environment }\end{array}$ & 82.50 & 6 & $\begin{array}{l}\text { Energy consumption per } \\
\quad \text { capita }\end{array}$ & 96.89 & 25 \\
\hline $\begin{array}{l}\text { Improved sanitation } \\
\text { facilities (\% of }\end{array}$ & 93.00 & 54 & $\begin{array}{l}5.2 \text { Economy and } \\
\text { Environment }\end{array}$ & 45.06 & 118 \\
\hline population with access) & & & Land resource utilization & 0.01 & 116 \\
\hline $\begin{array}{l}\text { Motor vehicles (per } 1,000 \\
\text { people) }\end{array}$ & 92.96 & 41 & $\begin{array}{l}\text { efficiency } \\
\text { Sulfur dioxide emissions }\end{array}$ & 81.31 & 111 \\
\hline $\begin{array}{l}\text { Renewable internal } \\
\text { freshwater resources } \\
\text { per capita }\end{array}$ & 10.74 & 36 & $\begin{array}{l}\text { per unit of GDP } \\
\text { Carbon dioxide emissions } \\
\text { per unit of GDP }\end{array}$ & 44.00 & 127 \\
\hline $\begin{array}{l}\mathrm{SO}_{2} \text { emissions (metric tons } \\
\text { per capita) }\end{array}$ & 97.84 & 44 & $\begin{array}{l}\text { Energy consumption per } \\
\text { unit of GDP }\end{array}$ & 54.91 & 117 \\
\hline
\end{tabular}

Table 80.2 Rank distribution of the individual indicators of GEC

\begin{tabular}{|c|c|c|c|c|c|c|}
\hline Sub-index & $\begin{array}{l}\text { Number } \\
\text { of the } \\
\text { individual } \\
\text { indicators }\end{array}$ & $\begin{array}{l}\text { Rank } \\
1-10\end{array}$ & $\begin{array}{l}\text { Rank } \\
11-30\end{array}$ & $\begin{array}{l}\text { Rank } \\
31-60\end{array}$ & $\begin{array}{l}\text { Rank } \\
61-100\end{array}$ & $\begin{array}{l}\text { Rank } \\
101-133\end{array}$ \\
\hline $\begin{array}{l}\text { Resource Environment } \\
\text { Competitiveness }\end{array}$ & 14 & 0 & 0 & 1 & 8 & 5 \\
\hline $\begin{array}{l}\text { Ecological Environment } \\
\text { Competitiveness }\end{array}$ & 11 & 1 & 2 & 1 & 6 & 1 \\
\hline $\begin{array}{c}\text { Environment Carrying } \\
\text { Competitiveness }\end{array}$ & 15 & 1 & 4 & 3 & 3 & 4 \\
\hline $\begin{array}{l}\text { Environment Management } \\
\text { Competitiveness }\end{array}$ & 10 & 0 & 2 & 2 & 3 & 3 \\
\hline $\begin{array}{c}\text { Environment Harmony } \\
\text { Competitiveness }\end{array}$ & 10 & 1 & 0 & 5 & 0 & 4 \\
\hline Total & 60 & 3 & 8 & 12 & 20 & 17 \\
\hline
\end{tabular}

Open Access This chapter is distributed under the terms of the Creative Commons Attribution Noncommercial License, which permits any noncommercial use, distribution, and reproduction in any medium, provided the original author(s) and source are credited. 